ORIGINAL ARTICLE

\title{
Measurement of movement is an objective method to assist in assessment of opiate withdrawal in newborns
}

\author{
C O'Brien, R Hunt, H E Jeffery
}

Arch Dis Child Fetal Neonatal Ed 2004;89:F305-F309. doi: 10.1136/adc.2002.025270

See end of article for authors' affiliations

....

Correspondence to: Dr Jeffery, Department of Neonatal Medicine, Royal Prince Alfred Hospital, Sydney 2050, New South Wales, Australia;

hieffery@med.usyd.edu.au

Accepted 22 October 2003

\begin{abstract}
Objective: To develop an objective and reliable method to assess drug withdrawal in newborns by quantitatively estimating the amount of movement rather than scoring individual withdrawal signs.

Design: In this cross sectional study, a commercial portable motion detector with computer memory, similar to a wrist watch (the actigraph) was used to measure movement. The measurements were compared with a clinical decision based on the neonatal abstinence syndrome (NAS) score. Movement was analysed, using non-parametric tests, in three groups: a control group of 10 infants, 13 opiate exposed newborns not treated for NAS, and 30 newborns treated for NAS (17 before treatment, eight within 24 hours of treatment, five when stabilised).

Results: There were significant differences in the median activity score, expressed as counts per minute (cpm), in the pretreatment group (124 cpm) compared with the control $(42 \mathrm{cpm}, \mathrm{p}<0.0001)$, nontreated $(74 \mathrm{cpm}, p=0.001)$, and stabilised treatment $(75 \mathrm{cpm}, p=0.007)$ groups. The accuracy of the actigraph in the identification of newborns requiring treatment from those who did not was high compared with the clinical scores; sensitivity $94 \%$; specificity $85 \%$; positive and negative predictive values $88 \%$ and $92 \%$ respectively.

Conclusions: The measure of movement is comparable to the clinical score in the identification of newborns who require treatment and in determining the severity of withdrawal. The clear advantage of this method is its objectivity, reliability, and efficiency as a simple, non-invasive, bedside measure. Further evaluation in a randomised, controlled trial would establish comparative benefits, potential harms, safety, and acceptability.
\end{abstract}

1: licit drug use during pregnancy is a major medical and social problem. One of the most important morbidities of opiate drug use during pregnancy is the infant withdrawal syndrome or neonatal abstinence syndrome (NAS), present in most newborns exposed in utero. ${ }^{1-4}$ NAS is defined as the physiological and behavioural state characterised by a series of withdrawal signs usually displayed within $48-72$ hours of birth. $^{5}$

In 1975, Finnegan and associates developed the assessment and treatment protocol for newborns called the NAS scoring system. ${ }^{67}$ The score is based on observation of over 30 clinical withdrawal signs and is repeated at regular intervals. The NAS scoring system has been used internationally for over 25 years, yet there remain unresolved and clinically important methodological problems.

Most literature on assessment and treatment of NAS was published before the widespread development of clinical epidemiology. ${ }^{8}$ The scoring method was validated in 1977 with sucking behaviour, although there is no clear evidence of a relation with severity of withdrawal and treatment requirements. ${ }^{9}$ Secondly, the scoring method is not a blinded assessment, can be prone to bias, and is often cumbersome. ${ }^{10}$ In a recent qualitative study of the principal users of the method, $44 \%$ of nurses reported that the scoring chart was not very useful, and 59\% indicated they would change something about the scoring system. ${ }^{11}$ There is a need for an objective measure in the assessment and management of NAS.

This paper describes the measurement of movement instead of scoring individual withdrawal signs. The concept of measuring movement was based on the assessment of four previous studies, documenting the incidence of individual withdrawal signs in a total of 334 newborns exposed to opiates in utero. ${ }^{12-15}$ We estimated that most newborns displayed withdrawal signs that were likely to be movement related-for example, irritability, restlessness, tremors, sneezing, regurgitation, frantic sucking, inability to sleep, and hyperactivity. This was supported by the documentation of sleep deprivation, disorganisation, fragmentation, and increased movement in newborns with NAS compared with controls. ${ }^{16}$ One other study found no significant difference in the amount of movement (measured by invasive needle electromyography) in heroin exposed newborns compared with control newborns, although movement was noted more often. ${ }^{17}$

The main aim of this study was to develop an objective, reliable, and efficient way to assess and manage NAS using a measure of movement. The specific objectives were to: (a) determine the amount of movement in healthy newborns and newborns with NAS and assess whether a commercial motion detector (actigraph) would be suitable and accurate enough to use in the postnatal wards and nurseries; $(b)$ compare the results of the computer recordings of movement with the clinical scoring system.

\section{METHODS}

\section{Subjects and selection criteria}

Newborn infants were selected from Royal Prince Alfred Hospital between April 2000 and January 2002 after ethics committee approval and informed written parental consent had been obtained.

Three newborn groups were selected as follows:

- Control group: 10 healthy newborns selected from the postnatal wards if 35-42 weeks gestation, no epidural or other drugs during delivery, no history of maternal drug dependency, and not admitted to the special care nursery or treated for jaundice. 
- No treatment group: 13 drug exposed newborns consecutively selected from the postnatal wards if 35-42 weeks gestation, a history of maternal opiate dependency (heroin or methadone) during the pregnancy, with or without polydrug use, and not admitted to the nursery for treatment of NAS.

- Treatment group: 27 drug exposed newborns consecutively selected if 35-42 weeks gestation, history of maternal opiate dependency (heroin or methadone) during the pregnancy, with or without polydrug use, and admitted to the nursery for treatment of NAS.

\section{Assessment and treatment of NAS}

Newborns were assessed using a modification of Finnegan's original scoring system designed in $1975^{67}$ (table 1). The attending nurse scored the 27 withdrawal signs every four hours, and, if three consecutive scores were 8 or above or two were 12 or above, the newborn was admitted to the nursery. Decisions on whether to treat (with oral morphine) were then made by an experienced paediatrician specialising in drug use in pregnancy and care of infants with NAS, according to protocol (http://www.cs.nsw.gov.au/rpa/ neonatal/default $2 / \mathrm{htm})$. The clinical and nursing staff were unaware of the actigraph readings before deciding whether treatment was required.

\section{Definitions for the treatment subgroups}

The treatment group was further subdivided into three subgroups according to the following definitions:

- Pretreatment: consecutively scoring $>8$ within the 24 hours leading up to treatment.

- Post-treatment: 24 hours after treatment had first started.

- Stabilised treatment: newborns scoring consecutively $<6$, feeding well, and gaining weight.

\section{Measurements of movement}

Movement was measured using a commercial motion detector called the actigraph. The recordings were made during the day and between feeds. A micro-mini-motionlogger actigraph (AMA-32; Ambulatory Monitoring, Ardsley, New York, USA) was placed on the newborn infant's left leg in accordance with previous studies that have validated and measured activity levels and sleep in newborn infants. ${ }^{18-21}$ The actigraph contains a programmable, microcomputer and a piezo-electric accelerometer. As the free end of the actigraph is moved, a voltage proportional to the mechanical deflection is generated. ${ }^{18-21}$ The zero crossing setting was used-that is, a count of activity was recorded each time the infant's leg moved, generating a voltage, which then crossed the preset threshold, with a filter setting mode of 18 defined by a bandpass of $2-3 \mathrm{~Hz}$. The activity score was then calculated, representing the average number of counts for each one minute epoch (cpm). A computer program, Action-W (Ambulatory Monitoring), developed by Sadeh and colleagues $^{18}{ }^{19}$ was used to analyse the activity scores.

\section{Clinical score}

In the no treatment and treatment groups, the recordings were examined during the corresponding period when nurses trained in the Finnegan scoring method completed the NAS chart (table 1). The predictive values of the actigraph measurement compared with the clinical decision incorporating the NAS score were calculated. ${ }^{22}$

\section{Statistical analysis}

Sample size was determined from a pilot study of 43 newborns, using a piezo-electrode to measure movement. ${ }^{23}$ The mean activity count was $38 \mathrm{cpm}$ for control infants and $110 \mathrm{cpm}$ for those requiring treatment, with a common standard deviation of $40 \mathrm{cpm}$. This established that a sample size of 10 in each group would have a power of $90 \%$ to detect a difference between newborns requiring treatment and

Table 1 Neonatal withdrawal scoring chart for term infants used at Royal Prince Alfred Hospital (modification of neonatal abstinence scoring system ${ }^{6}$ )

\begin{tabular}{|c|c|c|c|}
\hline System & Signs & Score & Date and Time in hours \\
\hline \multirow[t]{13}{*}{ Central nervous system disturbances } & High pitched cry & 2 & \\
\hline & Continuous high pitched cry & 3 & \\
\hline & Sleeps $<1$ hour after feeding & 3 & \\
\hline & Sleeps $<2$ hours after feeding & 2 & \\
\hline & Sleeps $<3$ hours after feeding & 1 & \\
\hline & Mild tremors, disturbed & 1 & \\
\hline & Mod-severe tremors, disturbed & 2 & \\
\hline & Mild tremors, undisturbed & 3 & \\
\hline & Mod-severe tremors, undisturbed & 4 & \\
\hline & Increased muscle tone & 2 & \\
\hline & Excoriation (specify area) & 1 & \\
\hline & Myoclonic jerks & 3 & \\
\hline & Generalised convulsions & 5 & \\
\hline \multirow{8}{*}{$\begin{array}{l}\text { Metabolic/ vasomotor/ respiratory } \\
\text { disturbances }\end{array}$} & Fever $\left(37.3-38.3^{\circ} \mathrm{C}\right)$ & 1 & \\
\hline & Fever (38.4 $4^{\circ} \mathrm{C}$ and higher) & 2 & \\
\hline & Frequent yawning ( $3-4$ times) & 1 & \\
\hline & Nasal stuffiness & 1 & \\
\hline & Sneezing ( $>3-4$ times) & 1 & \\
\hline & Nasal flaring & 2 & \\
\hline & Respiratory rate $>60 / \mathrm{min}$ & 1 & \\
\hline & Respiratory rate $>60 / \mathrm{min}$ with retractions & 2 & \\
\hline \multirow[t]{8}{*}{ Gastrointestinal disturbances } & Excessive sucking & 1 & \\
\hline & Poor feeding & 2 & \\
\hline & Regurgitation & 2 & \\
\hline & Projectile vomiting & 3 & \\
\hline & Loose stools & 23 & \\
\hline & Watery stools & 3 & \\
\hline & TOTAL SCORE & & \\
\hline & SCORER'S INITIALS & & \\
\hline
\end{tabular}


newborns not treated for NAS at a significance level of 0.01 . The basic data for each group were expressed as mean (SD; range) and compared using Student's $t$ test. A scatterplot was used to represent the non-parametric distribution for each group. As multiple comparisons were made, a conservative $p$ value of less than 0.01 was considered significant. The Kruskal-Wallis test was used to determine the differences in movement between the groups. If there was a significant difference, the Mann-Whitney U test was used to determine where the differences existed.

\section{RESULTS}

Overall, $82 \%$ of opiate exposed infants born at Royal Prince Alfred Hospital were recruited during the 22 month study period (eight were missed and the parents of one refused participation in the study).

\section{Subjects}

Table 2 shows the gestation, birth weight, centile, sex, postnatal day of study, and maternal drug use for the three groups. In the control group, there was no history of illicit drug use during pregnancy and no signs of NAS.

The mean (SD; range) NAS score at the time of the study was $4(3 ; 1-9)$ in the no treatment group and $8(3 ; 3-14)$ in the treatment group. Treatment started on average on day 3 ( $1 ; 1-4)$, and all newborns were treated with oral morphine. Recordings were made in 17 newborns before treatment, eight after treatment, and five when stabilized on treatment. Both treated and non-treated newborns with NAS had significantly lower birth weights than controls (table 2).

\section{Actigraph recordings of movement}

The total recording time for the control, no treatment, and treatment groups expressed as mean (SD; range) was 226 (117; 101-540), 294 (271; 73-870), and 353 (327; 55-1370) minutes respectively. The activity scores, expressed as a median (25th and 75th quartiles) for the control, no treatment groups, pretreatment, post-treatment, and stabilised groups were 42 (21-66), 74 (63-89), 124 (98-150), 88 (65-128), and 75 (43-90) cpm (fig 1). From these results, a treatment threshold was derived at $90 \mathrm{cpm}$. Significant increases in activity were evident in the no treatment $(p=0.0032)$, pretreatment $(\mathrm{p}<0.0001)$, and post-treatment $(\mathrm{p}=0.005)$ groups compared with the control group. A significant increase was also found in the pretreatment group $(\mathrm{p}=0.01)$ compared with the no treatment group.

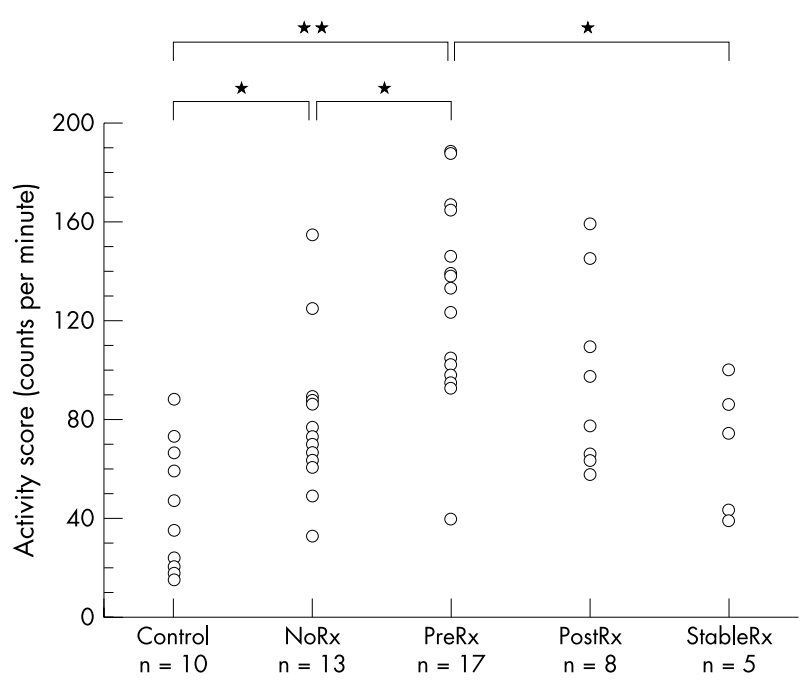

Figure 1 The activity count measured by the actigraph. The mean activity score for the control, no treatment (NoRx), pretreatment (PreRx), post-treatment (PostRx), and stabilised treatment (StabRx) groups is expressed as the number of counts per minute. There was a significant increase in activity in the pretreatment group $(124 \mathrm{cpm})$ compared with the control (42 cpm, $\left.{ }^{* *} p<0.0001\right)$, no treatment $\left(74 \mathrm{cpm},{ }^{*} \mathrm{p}=0.001\right)$, and stabilised $\left(75 \mathrm{cpm},{ }^{*} p=0.007\right)$ groups. Significant differences were also found in the no treatment $\left({ }^{*} p=0.009\right)$ and post-treatment $\left.l^{*} p=0.008\right)$ groups compared with the control group $~^{* *} p<0.001$; $\left.{ }^{*} \mathrm{p}<0.01\right)$.

\section{Actigraph and clinical score}

The sensitivity, specificity, and positive and negative predictive values were $94 \%, 85 \%, 88 \%$, and $92 \%$ respectively (table 3). Of the five newborns stabilised on treatment at the time of the study, all were correctly identified by measuring less than the treatment threshold.

\section{DISCUSSION}

Previous assessments of NAS have been limited to scoring methods, which are characteristically subjective, biased, and time consuming. The findings from this study confirm that the actigraph measurement of movement is comparable to the current clinical scoring method used at Royal Prince Alfred Hospital. In comparison with the traditional scoring method, movement is a continuous bedside measurement.

Table 2 Basic details of the control, no treatment, and treatment groups of newborns

\begin{tabular}{|c|c|c|c|}
\hline & Control $(n=10)$ & No treatment $(n=13)$ & Treatment $(n=27)$ \\
\hline Male:female & $5: 5$ & $6: 7$ & $17: 10$ \\
\hline Gestational age (weeks) & $39(1 ; 37-41)$ & $37(1 ; 35-39)^{*} \ddagger$ & $39(2 ; 35-41)$ \\
\hline Birth weight (g) & $3513(346 ; 3000-4020)$ & $2690(381 ; 2030-3255)^{*}$ & $2985(531 ;$ 2030-3880)† \\
\hline Centile & $59(23 ; 16-92)$ & $19(19 ; 1-61)^{*}$ & $30(25 ; 1-76) \dagger$ \\
\hline $\begin{array}{l}\text { Postnatal age at study } \\
\text { (days) }\end{array}$ & $3(1 ; 2-6)$ & $3(1 ; 2-5)$ & $3(2 ; 1-10)$ \\
\hline $\begin{array}{l}\text { Maternal history of drug } \\
\text { use }\end{array}$ & No & Yes & Yes \\
\hline Stable on methadone & & $46 \%$ & $48 \%$ \\
\hline $\begin{array}{l}\text { Using methadone and } \\
\text { heroin }\end{array}$ & & $15 \%$ & $11 \%$ \\
\hline Heroin only & & $23 \%$ & $8 \%$ \\
\hline $\begin{array}{l}\text { Using methadone, heroin } \\
\text { and other drugs }\end{array}$ & & $16 \%$ & $33 \%$ \\
\hline $\begin{array}{l}\text { Mean methadone dose } \\
\text { (mg) }\end{array}$ & & $55(29 ; 10-100)$ & $79(35 ; 13-180)$ \\
\hline \multicolumn{4}{|c|}{$\begin{array}{l}\text { Where applicable, values are mean (SD; range). } \\
\text { *Significantly different from the control group }(p<0.001) \text {. } \\
\text { tSignficantly different from the control group }(p<0.01) \text {. } \\
\text { tSignificantly different from the treatment group }(p=0.01) \text {. }\end{array}$} \\
\hline
\end{tabular}


Table 3 Accuracy of the actigraph in identifying treatment requirements for neonatal abstinence syndrome (NAS)

\begin{tabular}{|c|c|c|c|}
\hline \multirow[b]{2}{*}{ Actigraph activity mean } & \multicolumn{3}{|c|}{ Experienced clinical decision incorporating NAS score } \\
\hline & $\begin{array}{l}\text { Pretreatment ( } 3 \\
\text { scores }>8 \text { or one } \\
>12 \text { ) }\end{array}$ & $\begin{array}{l}\text { No treatment (consecutively } \\
\text { scoring }<8 \text { ) }\end{array}$ & $\begin{array}{l}\text { Stabilised treatment ( } 3 \\
\text { consecutive scores }<6 \text {, } \\
\text { feeding well and weight } \\
\text { gain) }\end{array}$ \\
\hline Greater than $90 \mathrm{cpm}$ & 16 true positive & 2 false positive & 0 \\
\hline Less than $90 \mathrm{cpm}$ & 1 false negative & 11 true negative & 5 \\
\hline
\end{tabular}

Sensitivity $94 \%$ (95\% confidence interval 71 to 99 ); specificity $85 \%$ (95\% confidence interval 55 to 98 ). cpm, Counts per minute

The most obvious advantage of the new method is its objectivity and reliability as a computer generated measure.

The first aim of this study was to determine the levels of movement in the three groups of newborns. The control group showed the range of movement that defined the healthy, normal pattern of activity. The no treatment group showed a higher level of movement than the control group and a lower level than the treatment group because most opiate exposed newborns show some withdrawal signs but these signs are not frequent enough or severe enough to warrant treatment.

The findings in the treatment group show how the measurement of movement can provide important clinical information for the assessment and management of NAS. There was a significant separation of the pretreatment group from the stabilised and no treatment groups (fig 1). This is consistent with this group representing "peak" withdrawal in the newborn as the recordings were conducted in the 24 hours leading up to treatment. This group also represented newborns with the greatest severity and frequency of withdrawal signs, and this was consistent with consecutively high scores that led to treatment with morphine.

An important feature of this method must be to indicate when doses of morphine can be reduced, to aid the assessment of when it is safe for the newborn to go home (other assessments include weight and feeding). Although longitudinal data are required to determine whether movement can identify when newborn infants are stabilised, the results so far suggest that movement in the stabilised group is reduced to a level between that of the control and no treatment groups (fig 1). These results can be explained by the effect of treatment, in this case morphine, on the withdrawal syndrome in the newborn. Morphine analogues occupy the opiate receptors in the central nervous system, autonomic nervous system, and gastrointestinal system and enable the newborn to function more normally by imposing a gradual rather than sudden withdrawal from the in utero dependency. The withdrawal signs diminish as a result of treatment, thus the clinical scores decrease, supporting the reduction in the amount of movement measured in this group.

Other differences included lower mean birth weight in the NAS groups than the control group, a finding observed in previous studies. ${ }^{24-27}$

The second aim of this study was to compare the results of the polygraphic recordings of movement with the clinical scoring system. The accuracy of the actigraph measurement as a clinical diagnostic tool was examined using the current widely used "standard" to identify newborns requiring treatment for NAS-that is, an experienced clinician interpreting the clinical score. The sensitivity and specificity of the movement measurement was high $(\geqslant 85 \%)$, indicating that it can distinguish between newborns who require treatment from those who do not, with high accuracy compared with the current scoring method. As there is no ideal method for the assessment and treatment of NAS, it was not possible to determine if movement is a more accurate measure of neonatal drug withdrawal than the current method.

Further modification, based on the one false negative and two false positive results, suggests that predictive values would approach $100 \%$ accuracy. The one false negative measurement in the pretreatment group occurred in an infant with predominantly non-movement related withdrawal signs including poor feeding, increased muscle tone, and watery stools. ${ }^{12-15}$ Although an uncommon presentation, for clinical application, the measure will have to be used in conjunction with observation of feeding scores and stool type, and the clinician will need to include these observations in the decision making process.

The reasons for the two false positive measurements raise important problems. Firstly, unsettled periods are important to measure but can arise falsely from the mother's movement when holding her newborn for extended periods of time while on the postnatal ward, even though the baby may be asleep and settled. This needs discussion with parents in order to minimise artefacts. Other potential manipulation, such as removal of the actigraph, is readily detected by prolonged zero movement level marked in red on the trace.

The second reason for a false positive measurement concerns activity around the chosen treatment threshold of $90 \mathrm{cpm}$. Newborns not treated for NAS often show periods of high activity followed by decreased activity, whereas newborns who require treatment show trends of increasing activity between feeds. From the results, high activity counts (greater than $120 \mathrm{cpm}$ ) indicate the need for treatment, and low activity counts (less than $80 \mathrm{cpm}$ ) indicate no need for treatment. To determine treatment requirements for newborns with $80-120 \mathrm{cpm}$, we suggest monitoring for another four hours; if movement increases, treatment is required, and if movement decreases, treatment is not required.

The measurement of movement is time saving compared with the clinical score especially in the postnatal ward where the aim is to keep mothers and babies together safely. Often, there is only one nurse to a minimum of four mothers and babies, which makes the accurate monitoring of withdrawal signs very difficult, and the scorer changes in eight hourly shifts. In comparison, the actigraph takes one minute to place on the leg and can be left in situ for the recording period. Five minutes is required to complete the analysis on the computer at a chosen location.

In conclusion, this study shows that the measurement of movement is comparable to the clinical score in determining the severity of withdrawal and treatment requirements in newborns with NAS. The clear advantage of this method is its objectivity, reliability, and efficiency as a simple, noninvasive, bedside measure. Further evaluation in a randomised, controlled trial, using the actigraph in conjunction with observation of non-movement related withdrawal signs compared with the current clinical scoring method, would 
establish comparative benefits, potential harms, safety, and acceptability.

\section{ACKNOWLEDGEMENTS}

We thank the mothers and newborn infants for their participation in this study, and the staff in the postnatal wards and special care nurseries for their assistance in the recruitment of newborns and for their support and interest. We gratefully acknowledge George McKelvey for his technical support, and Dr Crista Wocadlo and Dr David Osborn for their statistical assistance.

\section{Authors' affiliations}

C O'Brien, R Hunt, H E Jeffery, Department of Neonatal Medicine, Royal Prince Alfred Hospital, Sydney, Australia

C O'Brien, H E Jeffery, University of Sydney

\section{REFERENCES}

1 Madden J, Chappel J, Zuspan F, et al. Observation and treatment of neonatal narcotic withdrawal. Am J Obstet Gynecol 1977;127:199-201.

2 Lam S, To W, Duthie S, et al. Narcotic addiction in pregnancy with adverse maternal and perinatal outcome. Aust N Z J Obstet Gynaecol 1992;32:216-21.

3 Householder J, Hatcher R, Burns W, et al. Infants born to narcotic-addicted mothers. Psychol Bull 1982;92:453-68.

4 Dawkins J, Tylen E, Colley N, et al. Drug abuse in pregnancy: obstetric and neonatal problems. Ten years' experience. Drug Alcohol Rev 1997; 16:25-31

5 Suresh S, Anand K. Opioid tolerance in neonates: mechanisms, diagnosis, assessment, and management. Semin Perinatol 1998;22:425-33.

6 Finnegan L, Kron R, Connaughton J, et al. Assessment and treatment of abstinence in the infant of the drug-dependent mother. Int J Clin Pharmacol Biopharm 1975;12:19-32.

7 Finnegan L, Mitros T, Hopkins L. Management of neonatal narcotic abstinence utilizing a phenobarbital loading dose method. NIDA Res Monogr 1979;27:247-53.

8 Kron R, Finnegan L, Kaplan S, et al. The assessment of behavioral change in infants undergoing narcotic withdrawal: comparative data from clinical and objective methods. Addict Dis 1975;2:257-75.

9 Theis JGW, Selby P, Ikizler Y, et al. Current management of neonatal abstinence syndrome: a critical analysis of the evidence. Biol Neonate 1997;71:345-56.
10 Zahorodny W, Rom C, Whitney W, et al. The neonatal withdrawal inventory: a simplified score of newborn withdrawal. J Dev Behav Pediatr 1998;19:89-93.

11 Hulatt J. Neonatal abstinence syndrome: how and where should babies with this condition be cared for? Journal of Neonatal Nursing 2000;6:159-64.

12 Finnegan L, Connaughton JJ, Kron R, et al. Neonatal abstinence syndrome: assessment and management. Addict Dis 1975;2:141-58

13 Ostrea EJ, Chavez C, Strauss M. A study of factors that influence the severity of neonatal narcotic withdrawal. Addict Dis 1975;2:187-99.

14 Rosen T, Pippenger C. Pharmacologic observations on the neonatal withdrawal syndrome. J Pediatr 1976;88:1044-8.

15 Harper R, Solish G, Feingold E, et al. Maternal ingested methadone, body fluid methadone, and the neonatal withdrawal syndrome. Am J Obstet Gynaecol 1977; 129:417-24.

16 O'Brien CM, Jeffery HE. Sleep deprivation, disorganisation and fragmentation during opiate withdrawal in newborns. J'Paediatr Child Health 2002;38:66-71.

17 Schulman C. Alterations in sleep cycle in heroin and suspect newborn. Neuropediatrie 1969;1:89-100.

18 Sadeh A, Aceob C, Seifer R, et al. Activity-based assessment of sleep-wake patterns during the 1 st year of life. Infant Behaviour and Development 1995; 18:329-37.

19 Sadeh A Alster J, Urbach D, et al. Actigraphically based automatic bedtime sleep-wake scoring: validity and clinical applications. Journal of Ambulatory Monitoring 1989;2:209-16.

20 Sadeh A, Lavie P, Scher A, et al. Actigraphic home monitoring sleep-disturbed and control infants and young children: a new method for pediatric assessment of sleep-wake patterns. Pediatrics 1991:87:494-9.

21 Mennella J, Gerrish C. Effects of exposure to alcohol in mother's milk on infant sleep. Pediatrics 1998;101:e2.

22 Sackett D, Haynes R, Guyatt G, et al. Clinical epidemiology: a basic science for clinical medicine, 2nd ed. Boston: Little, Brown and Company, 1991:19-51.

23 O'Brien CM, Jeffery HE. Measuring movement: a more objective, reliable and efficient method to assess drug withdrawal in newborns. 5th Annual Congress Proceedings of the Perinatal Society of Australia and New Zealand, Canberra, March, 2001.

24 Kelly JJ, Davis PG, Henschke PN. The drug epidemic: effects on newborn infants and health resource consumption at a tertiary perinatal centre. J Paediatr Child Health 2000;36:262-4.

25 Olofsson M, Buckley W, Andersen GE, et al. Investigation of 89 children born by drug-dependent mothers. I. Neonatal course. Acta Paediatric Scand 1983;72:403-6.

26 Zelson C, Rubio E, Wasserman E. Neonatal narcotic addiction: 10 year observation. Pediatrics 1971;48:178-89.

27 Oleske JM. Experiences with 118 Infants born to narcotic using mothers. Clin Pediatr 1977;16:418-23. 